\title{
RFID-Based Wireless Multi-Sensory System for Simultaneous Dynamic Acceleration and Strain Measurements of Civil Infrastructure
}

\author{
Devaka Jayawardana, Member, IEEE, Ranjith Liyanapathirana, Senior Member, IEEE, \\ and Xinqun Zhu
}

\begin{abstract}
In this paper, we develop a radio frequency identification (RFID)-based wireless multi-sensory infrastructure health monitoring (IHM) system that can simultaneously measure dynamic acceleration and strain. The system consists of a novel multi-sensor integrated semi-passive ultra-high frequency (UHF) tag antenna that can be mounted on civil infrastructure elements; even made out of metal. The system is capable of measuring 3-axis dynamic acceleration and strain with spectral bandwidths of $40 \mathrm{~Hz}$ and $26.5 \mathrm{~Hz}$, respectively. The natural frequency determination of infrastructure by the dynamic acceleration and strain measurements of the proposed system is accurate to $60 \mathrm{mHz}$. Benchmarking of the RFID-based wireless multi-sensor system is provided by comprehensive comparison of the results with measurements from a commercial wireless strain measurement system. The proposed system has $30 \mathrm{mHz}$ natural frequency determination error when compared with dynamic strain measurement from the commercial system.
\end{abstract}

Index Terms- Acceleration, dynamic measurements, infrastructure health monitoring, RFID, strain, structural health monitoring

\section{INTRODUCTION}

A SSESSMENT of the prevailing condition of critical infrastructure facilities such as bridges and buildings is vital since a failure of such facilities would result-in loss of human life, property and millions of dollars of investment. Monitoring infrastructure using non-structure sensor systems in order to predict their health is known as infrastructure health monitoring (IHM) [1]. The modern trend is to use wireless sensor systems to avoid high installation costs and inconveniences inherent to wired sensor systems [2]. Acquisition of dynamic acceleration and strain is ubiquitous in IHM in order to generate rich information such as natural frequencies and mode shapes related to infrastructure under assessment. The natural frequencies of most large-scale civil structures are spread within the band of $0 \mathrm{~Hz}$ to $10 \mathrm{~Hz}[3,4]$.

Acquisition of dynamic acceleration measurements has

D. Jayawardana was with the Centre for Infrastructure Engineering, School of Computing, Engineering and Mathematics, Western Sydney University, Penrith, NSW 2751 Australia. He is now with the Ceylon Electricity Board, Colombo, Sri Lanka. (e-mail: ajydm@yahoo.com).

R. Liyanapathirana is with the School of Computing, Engineering and Mathematics, Western Sydney University, Penrith, NSW 2751 Australia. (email: r.liyanapathirana@westernsydney.edu.au).

X. Zhu is with University of Technology, Sydney (e-mail: Xinqun.Zhu@uts.edu.au) been done at numerous previous occasions for IHM of various civil structures [5-8]. Acceleration measurements can only provide global sense of the infrastructure as they are less sensitive in identification of local damage. On the contrary, strain directly relates to deformation of infrastructure and highly sensitive for local damage $[9,10]$. The mode curvature acquired by dynamic strain measurements, strain frequency response and strain energy are used to evaluate fault scenarios of structures. The use of dynamic strain measurements to derive those features has been performed in $[9,11,12]$. The importance of multiple measurands has been highlighted in $[14,15]$ for multimetric sensing-based IHM. In particular, it has been specified that combined consideration of dynamic responses of both acceleration and strain would provide more accuracy and reliability $[14,16]$ in IHM. Often, the strain and acceleration are measured by dedicated systems [16-18]. However, simultaneous measurement of important measurands such as strain and acceleration by a single system is advantageous in filtering out infrastructure state perturbations related to environmental condition changes [1921], and negate the effect of different system quality factors (i.e., error, accuracy) for IHM. Also, both global and local information related to infrastructure can be acquired using a single sensor. In order to cater such requirement, there are instances where multiple measurements were performed using active sensor nodes which consumes power for wireless data transfer [22,23].

RFID technology is attractive for low power wireless sensor development due to its inherent passive communication. Possible low power sensor development with RFID can be passive or semi-passive in which former does not contain a power source while latter contains, but does not consume its power for wireless data transfer [24,25]. Therefore, both of these RFID sensor types overcome the major drawback of active wireless sensors which is the consumption of power for wireless data transfer. Passive RFID sensors have the vital advantage of no battery requirement over semi-passive RFID sensors. Passive RFID sensors can simply be tag antennas or sensor integrated tag antennas. A meander-line dipole tag antenna has been investigated as a strain sensor for IHM applications by relating backscatter signal power measurements to static strain [26]. The resonant frequency shift of a folded patch tag antenna has been related to static strain in [27]. A dipole tag antenna has been studied to monitor the displacement of a metal beam by relating both 
transmit and backscatter signal power measurements to amount of displacement [28]. The crack widening detection sensor presented in [29] was consisted of two closely coupled dipole tag antennas. The phase change of the backscattered signal from the both RFID tag antennas due to increasing inter-tag antenna distance is correlated with crack widening. To the disadvantage of above sensors, the indirect raw measurements of backscatter signal power, reader transmit power and the resonant frequency can be highly peculiar in real infrastructure environments with multiple concrete and metal structural members with different orientations. Sensor integrated tag antennas do not have above disadvantages as they acquire the direct measurement of required physical quantity (i.e., acceleration, strain, etc.). Strain measurements related to IHM by RFID tag antenna integrated strain gauges were previously reported in [30,31]. A corrosion sensor integrated tag antenna to monitor reinforcement of concrete structures has been studied in [32]. Concrete maturity and status monitoring have been performed by temperature and humidity sensor integrated tag antennas in [33] and [34], respectively. However, the capability of continuous monitoring for dynamic information acquisition was not shown in above investigations. This is mostly due to insufficient power available for sensor integrated tag antennas in order to carryout continuous measurements as they only use harvested receive signal power from reader at their RF frontend.

When considering dynamic measurements, semi-passive RFID sensors have advantage over passive RFID sensors since the required power for continuous measurement can be drawn from the battery. A semi-passive RFID-based strain sensor presented in [35] for wireless IHM had the capability of measuring dynamic strain. The strain gauge sensor consisted of resistance-to-frequency conversion electronics and two dipole antennas. One antenna is used for modulated backscattering of identification information and for the waking up the sensor. The other antenna is used for backscattering of the output from resistance to frequency conversion electronics. The sensor has also shown a good read range. A commercial RFID-based strain measurement system with battery-assisted sensors is presented in [36].

Multiple sensor integration with RFID tag antenna was achieved using an augmented sensor module for environmental monitoring [37] consisting of acceleration, light and temperature sensors. Such multiple sensor integration approaches also investigated in [38] for temperature and acceleration measurements and in [32] for corrosion and acceleration. However, the possibilities of measuring dynamic acceleration were not shown. or the sensors' mounting capability on metal structures.

Therefore, the availability of RFID-based wireless systems which can simultaneously measure dynamic acceleration and strain of civil infrastructure is limited. The work presented in this paper is predominantly concentrated on filling this gap by introducing a multi-sensor integrated semi-passive RFID tag antenna-based wireless system to acquire multiple dynamic measurands. Developed tag antenna-based sensor can be directly mounted on civil structures; even made out of metal. Section II of this paper describes the RFID-based wireless multi-sensor system in detail; including the hardware and firmware designs. The measurement results comprising calibration and simultaneous dynamic acceleration and strain values are provided in Section III. System benchmarking with a commercial wireless strain measurement system is done in Section IV while the conclusions are given in Section V.

\section{RFID-BASED WIRELESS MULTI-SENSORY SYSTEM}

The multi-sensory system presented in this paper is capable of simultaneously measuring both dynamic acceleration and strain. The experimental setup for a cantilever steel beam is shown in Fig. 1(a). It has a novel multi-sensor integrated tag antenna (MSITA), Impinj Speedway Revolution UHF RFID reader, Laird Technologies S9025PR circularly polarized reader antenna having maximum gain of $5.5 \mathrm{dBi}$ and a laptop computer with a developed data acquisition application software. The reader has $-80 \mathrm{dBm}$ sensitivity, $31.5-\mathrm{dBm}$ maximum transmit power and the capability of handling multiple reader antennas. Furthermore, the reader provides convenience in developing data acquisition applications with Octane software development kit (SDK). The detailed description of the hardware setup, data acquisition and firmware design details is provided in following sections.

\section{A. Hardware Design of Multi-Sensor Integrated Semi- Passive Tag Antenna}

The MSITA is shown in Fig. 1(a) and illustrated in detail in Fig. 2. The integrated unit includes the construction material mountable RFID tag antenna designed and investigated in [39] and a custom designed multi-sensor circuit, which includes a 3 -axis accelerometer and a strain gauge, as shown in Fig. 2. The tag antenna is integrated with the multi-sensor circuit via the $\mathrm{I}^{2} \mathrm{C}$ interface. Ultra-low-power, 3-axis MEMS accelerometer ADXL362 from ANALOG DEVICES was used in the multi-sensor circuit. It has firmware configurable output data rates, noise modes, and maximum acceleration levels achieved via a 4-wire serial peripheral interface (SPI), and also it provides 3 -axis acceleration with 12-bit resolution via the same SPI. Furthermore, the selected accelerometer has configurable interrupts for different states and a first-in-firstout (FIFO) buffer. Additional hardware required to interface a commercial $120-\Omega$ strain gauge from TML in a 3 -wire quarter bridge configuration was provided in the design and fabrication stage. This type of strain gauge is suited to be easily attached to both concrete and metal surfaces. In the current design, the strain gauge is wired to the circuit taking into account the general concept that it is less probable to sense both dynamic acceleration and strain at the same position of a structure. Therefore, the 3 -wire configuration was provided as it introduces any resistance related to lead wires to both sides of the Wheatstone bridge (onboard; consisting $120-\Omega$ precision resistors from VISHAY with lowtemperature coefficient). Therefore, the lead wire resistance perturbation influence on quarter bridge voltage $\left(V_{B}\right)$ is minimum [40]. 


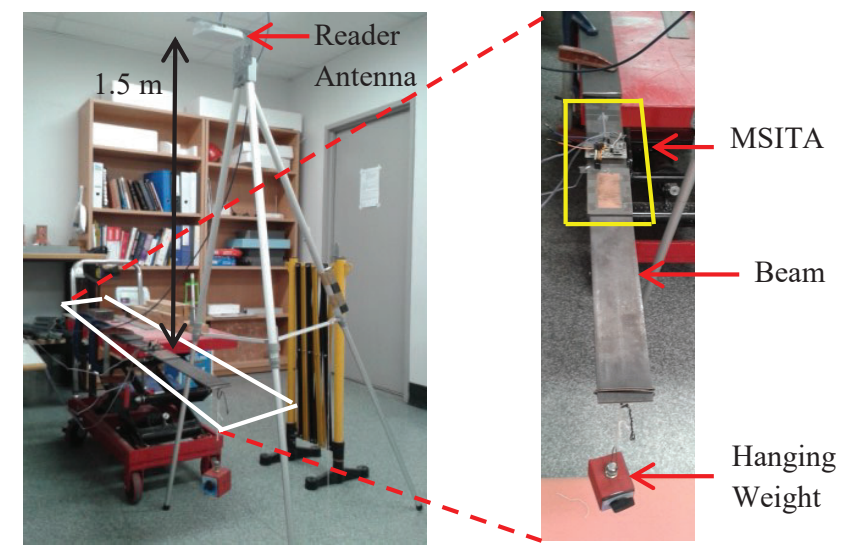

(a)

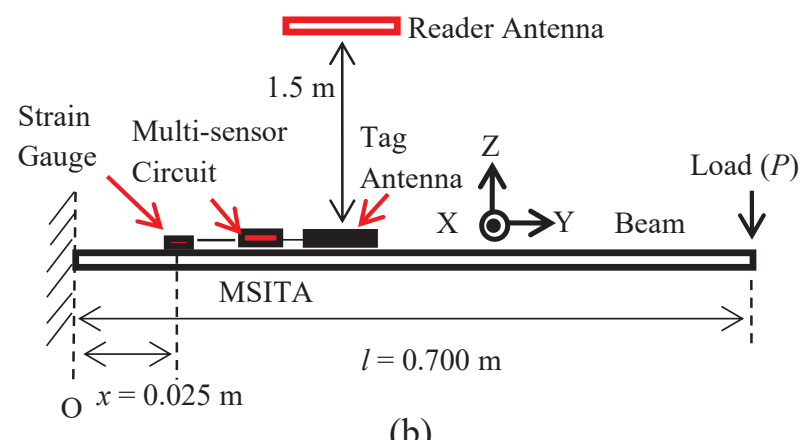

(b)

Fig. 1. RFID-based wireless multi-sensory system measuring dynamic acceleration and strain of cantilever steel beam: (a) laboratory setup and (b) schematic diagram.

A Texas Instruments (TI) INA333 instrumentation amplifier was used as the differential amplifier and it provides a lowpower, low-noise package with a high common-mode rejection ratio (CMRR). Furthermore, it has inbuilt radio frequency interference filters for each input which would help to filter noise induced by RF interference (in particular when the strain gauge is used with lead wires).

MSITA includes a programmable gain amplifier (PGA) which was introduced for two purposes. One is to increase the gain of the strain gauge interface in the multi-sensor circuit. The preceding differential amplifier has a maximum gain of $30 \mathrm{~dB}$. With the introduction of the Microchip MCP6S91 programmable gain amplifier, MSITA can reach maximum gain of $45 \mathrm{~dB}$ in combination with the differential amplifier. The second purpose is to provide the gain changing flexibility to the firmware of the multi-sensor circuit without any hardware alterations. The differential amplifier gain could only be altered by changing the value of the gain resistor [41]. The gain alteration can be performed by configuring the PGA via the 3-wire SPI. The filter was designed as a single pole, unity-gain, low pass filter which has a $50-\mathrm{Hz}$ cut-off frequency using a TI low power single supply OPA333 operational amplifier.

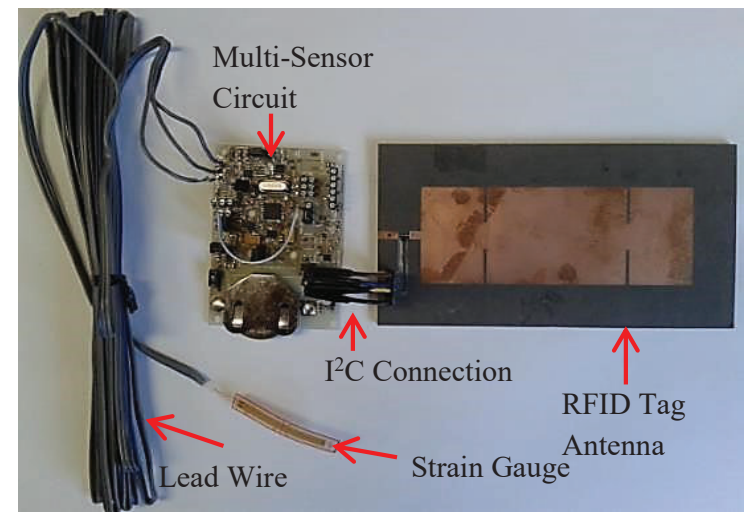

Fig. 2. Multi-sensor integrated tag antenna (MSITA).

The 16-bit PIC24F16KA102 ultra-low-power microcontroller was used in the multi-sensor circuit for fast handling of acceleration and strain data with 12-bit and 16-bit resolutions, respectively. It has a built-in 10-bit analog to digital converter (ADC) module with 9 channels. One of these channels was directly used for digitization of the strain signal. Since the strain signals related to large scale infrastructure are always expected to be small in magnitude, an extra 16-bit ADC channel was also dedicated to acquiring higher resolution information via the strain interface of the multisensor circuit. This was accomplished by introducing an ANALOG DEVICES AD7680 16-bit low power ADC integrated circuit. The extended 3-wire SPI bus was used to acquire higher-resolution 16-bit $\mathrm{ADC}$ strain data to the microcontroller.

Owing to different stages of amplification, filtering, and $\mathrm{ADC}$, the multi-sensor circuit can be used in different configurations. Each of these stages was isolated and the provision for the selection was provided by jumpers of selection switches introduced in the multi-sensor circuit. Isolation was primarily done to provide full configuration flexibility for the circuit when it is being used with different scenarios to measure dynamic acceleration and strain of structures. Additionally, it has assisted testing and calibration of the circuit prior to taking measurements. The multi-sensor circuit is powered by Panasonic CR2450 3-V coin cell having 650-mAh capacity.

\section{B. Firmware Design of Multi-Sensor Integrated Semi-Passive Tag Antenna}

The firmware of the MSITA was designed with the necessary requirements to simultaneously acquire strain and 3axis acceleration measurements. Since the MSITA is a semipassive, sensor-integrated tag antenna, the wireless communication is always initiated by the RFID reader. The Impinj Monza -X Dura 8k RFID chip used to design the tag antenna has operating states of "Internal Control" and "I2C Control" that has precedence over "Idle and RF Receive" state in which the reader is permitted to interrogate the tag chip [42]. The tag chip is in Internal Control state when data are written to its non-volatile (NVM) memory. Therefore, the tag chip is inaccessible via the RF air interface during the $\mathrm{I}^{2} \mathrm{C}$ data transfers between itself and the microcontroller. The 
maximum number of 16-bit words that can be acquired by the reader at a given time was limited to 64 , due to the use of "Optimized Tag Read" method in the data acquisition application based on Octane SDK. Moreover, an adequate time window to acquire both the dynamic acceleration and the strain measurements according to the data packet shown in Fig. 3 via the air interface was created in the firmware. In the formulation of the data packet, the reader accesses both "EPC" and "USER" memories of the tag chip. The electronic product code (EPC) can be configured to have 1 to 8 numbers of words (The EPC is currently configured to 2 words). It has been used to uniquely identify the MSITA in the data acquisition application which creates a text file that includes data from the USER memory. $\mathrm{T}_{\mathrm{W}}$ is the tracking word which was used to filter out multiple acquisitions that take place within the provided time window. $\mathrm{T}_{\mathrm{W}}$ has been toggled between two known values by the firmware for every $\mathrm{I}^{2} \mathrm{C}$ transaction between the microcontroller and the tag chip.

In the process of creating the time window for the reader, the accelerometer was configured to operate in the measurement mode, and a series of 45 acceleration data samples were allowed to accumulate at $80 \mathrm{~Hz}$ output data rate (ODR) inside the FIFO buffer of the accelerometer. Consequently, upon the "Watermark" interrupt generated by the accelerometer, the RF ports of the tag chip were blocked (end of time (EOT) window) and the acceleration samples were transferred to the microcontroller. The strain samples were acquired with a $53-\mathrm{Hz}$ sampling rate while both sample types were transferred to the USER memory of the tag chip. Finally, the RF ports were unblocked (start of time (SOT) window) to make the tag chip memory available to the reader for read operations via the air interface.

The firmware level power saving features of the MSITA were enabled by letting it run at a clock rate of $2 \mathrm{MHz}$ and implementing the Deep Sleep mode of the microcontroller.

The instruction cycle clock was used to derive the 2-MHz SPI clock, the $400-\mathrm{kHz} I^{2} \mathrm{C}$ clock and the $41-\mathrm{kHz}$ pulse width modulation (PWM - 50\% duty cycle) signal as the external clock for the accelerometer. The Deep Sleep mode of the PIC24F16KA102 microcontroller was implemented using "Write Wake Up" feature of the tag chip as the wake-up source. Therefore, the MSITA can be wirelessly woken up by the RFID reader. After waking up from the Deep Sleep mode, the MSITA goes into the measurement mode for both acceleration and strain for a pre-set time within which the reader acquires the measurements. The MSITA goes back to Deep Sleep at the end of the pre-set time for measurements. The firmware configuration parameters and set values for the MSITA is given in Table I.

\section{MEASUREMENT RESUlTS}

As shown in Fig. 1(a), the RFID-based wireless multisensor system was used to measure dynamic acceleration and strain of a cantilever steel beam. The cantilever portion of the beam has a length $(l)$ of $0.700 \mathrm{~m}$, width $(b)$ of $0.065 \mathrm{~m}$ and height $(h)$ of $0.005 \mathrm{~m}$. The MSITA was mounted on the beam as depicted in the expanded view of Fig. 1(a). The strain gauge is installed following a standard attachment method for metal surfaces at a distance $(x)$ of $0.025 \mathrm{~m}$ from O. Fig. 1(b) provides the schematic diagram of the cantilever beam measurement setup. The loading of the beam was done at the free end of the beam with hanging weights as shown in the expanded view of Fig. 1(a). The RFID reader has been set to Max Throughput mode from the data acquisition application written in Octane SDK. In this reader mode, the read rate depends upon the sensitivity, interference and environment. The calibration and strain measurement process is described next.

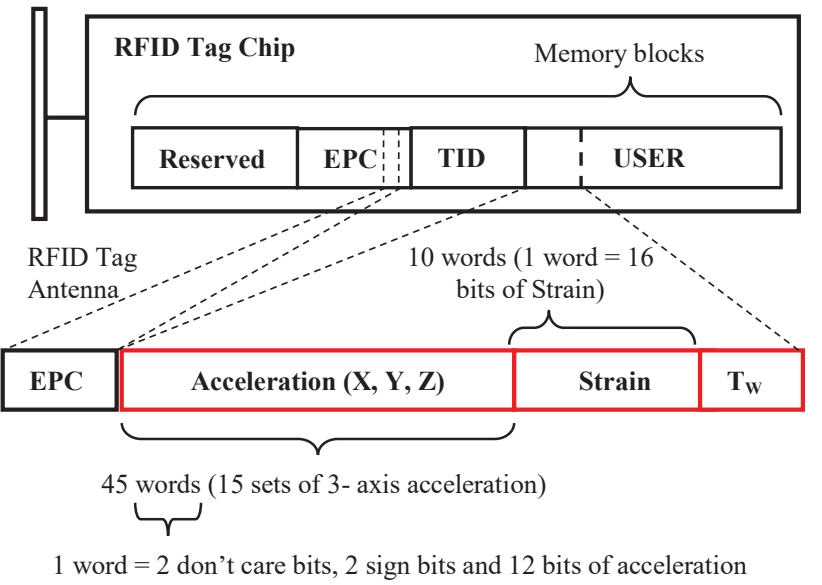

Fig. 3. Data packet containing 3-axes acceleration and strain (EPC-Electronic Product Code and $\mathrm{T}_{\mathrm{W}}$-tracking word).

TABLE I

CONFIGURATION OF COMPONENTS IN FIRMWARE

\begin{tabular}{|c|c|c|}
\hline \multirow{2}{*}{ Component } & \multicolumn{2}{|c|}{ Configuration } \\
\hline & Parameters & Set Values \\
\hline Accelerometer & $\begin{array}{l}\text { Acceleration level } \\
\text { Clock } \\
\text { ODR selected } \\
\text { ODR actual } \\
\text { Noise Mode } \\
\text { Interface } \\
\text { Anti-aliasing filter } \\
\text { Interrupt }\end{array}$ & $\begin{array}{l}2 \mathrm{~g} \\
\text { External }(41 \mathrm{kHz}) \\
100 \mathrm{~Hz} \\
80 \mathrm{~Hz} \\
\text { Ultra-low noise } \\
\text { 4-wire SPI (Slave) } \\
\text { ODR/2 } \\
\text { Watermark configured at } 45 \\
\text { acceleration samples }\end{array}$ \\
\hline $\begin{array}{l}\text { RFID Tag } \\
\text { Antenna }\end{array}$ & $\begin{array}{l}\text { RF ports } \\
\text { Interface }\end{array}$ & $\begin{array}{l}1 \text { blocked, } 2 \text { in use (blocked } \\
\text { when necessary) } \\
\mathrm{I}^{2} \mathrm{C} \text { (slave) }\end{array}$ \\
\hline Microcontroller & $\begin{array}{l}\text { Interfaces } \\
\text { Oscillator } \\
\text { 10-b ADC }\end{array}$ & $\begin{array}{l}\mathrm{I}^{2} \mathrm{C} \text { (master) } 400 \mathrm{kbps}, \mathrm{SPI} \\
\text { (master) } 2 \mathrm{MHz} \\
\text { Primary (4-MHz crystal } \\
\text { oscillator) } \\
\text { AN1[41] }\end{array}$ \\
\hline $\begin{array}{l}\text { Differential } \\
\text { Amplifier }\end{array}$ & $\begin{array}{l}\text { Gain resistance } \\
\text { Reference voltage }\end{array}$ & $\begin{array}{l}200 \Omega(\text { Gain }=500) \\
1.25 \mathrm{~V}\end{array}$ \\
\hline PGA & Interface & $\begin{array}{l}1 \text { (i.e., adequate gain has } \\
\text { been achieved through } \\
\text { Differential Amplifier) } \\
\text { 3-wire SPI (slave) }\end{array}$ \\
\hline Filter & $\begin{array}{l}\text { Type } \\
\text { Gain } \\
\text { Cut-off frequency }\end{array}$ & $\begin{array}{l}\text { Single pole low pass } \\
1 \text { (unity) } \\
50 \mathrm{~Hz}\end{array}$ \\
\hline 16-b ADC & Interface & 3-wire SPI (slave) \\
\hline
\end{tabular}




\section{A. Static Strain Measurements and Calibration of Strain Interface}

In this experiment, the strain measurement of the position shown in Fig. 1(b) was acquired by discretely increasing the load in similar amounts $(\sim 10 \mathrm{~N})$. In addition to the MSITA, a commercial wireless strain sensor system (CWLSS) and a standard wired digital strain meter (DSM) listed in Table II were used to carry out the same measurement. These systems were used with the same type of strain gauge as with the MSITA. Fig. 4 depicts the measurement results along with the theoretically calculated strain given by

$$
\epsilon=\frac{6 P(l-x)}{E b h^{2}}
$$

bending moment formula related to Fig. 1(b) where $\epsilon$ is the strain, $P$ is the load $(\mathrm{N})$ and $E$ is the Young's modulus $\left(\mathrm{Nm}^{-2}\right)$. The default offset of each system was removed from the measurement results. The root mean square error (RMSE) values were calculated using linear regression for the measurement results from each system. According to Fig. 4, the DSM has the results much close to the theoretical values. The results of MSITA are close to that of DSM. The CWLSS has the highest linearity (comparing RMSE) in the tested loading range mostly. The measurement results from the MSITA are non-linear as the relationship between quarter bridge voltage $\left(V_{B}\right.$ in $\left.\mathrm{V}\right)$ and $\epsilon$ as given by

$$
\frac{V_{B}}{V_{I}}=\frac{K \epsilon}{4}\left(\frac{1}{1+K \frac{\epsilon}{2}}\right),
$$

where $K$ is the gauge factor and $V_{I}$ is the quarter bridge input voltage $(\mathrm{V})$. The linearity of the results is close to that of the DSM in the strain range. Since the DSM strain values are almost equal to the theoretical strain values, it was chosen as the benchmark to calibrate both the MSITA and the CWLSS systems. In the process of calibrating MSITA and CWLSS, $K$ values of 2.03 and 1.82 which have been found by static strain measurements were used, respectively.

\section{B. Simultaneous Measurement of Tri-Axis Dynamic Acceleration and Strain by MSITA}

Simultaneous measurement of the tri-axis dynamic acceleration and strain were conducted upon the same cantilever beam shown in Fig. 1(a). An initially stable hanging load of $10.10 \mathrm{~N}$ was suddenly released to induce the free vibration of the beam. Fig. 5 depicts the time history of the dynamic acceleration measurement results along $Z$-axis and the related dynamic strain measurement. The $Z$ - axis has the highest acceleration as the force to induce vibration was exerted in the same direction. Both $X$ and $Y$ axes also showed small amounts of acceleration. Figs. 6(a) and 6(b) provide the single-sided amplitude spectra calculated from the tri- axis dynamic acceleration measurement and the strain measurement, respectively.
TABLE II

\begin{tabular}{|c|c|}
\hline System & Components \\
\hline CWLSS & $\begin{array}{l}\text { LORD MicroStrain SG-Link_LXRS wireless analog } \\
\text { 2-channel sensor node } \\
\text { LORD MicroStrain WSDA -Base-104-LXRS base } \\
\text { station }\end{array}$ \\
\hline DSM & TC-31K Digital Strain Meter from TML \\
\hline
\end{tabular}

SYSTEMS USED IN THE CALIBRATION

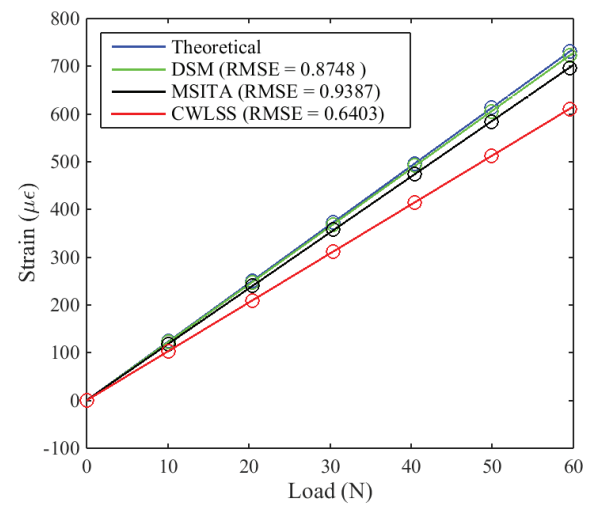

Fig. 4. Static strain measurement results (RMSE-root mean square error)

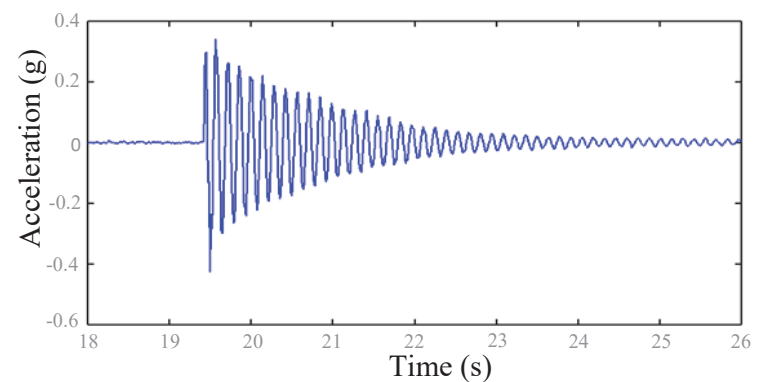

(a)

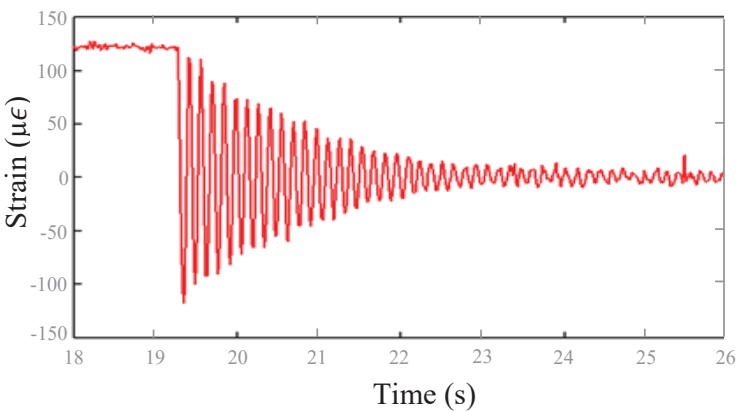

(b)

Fig. 5. Time history of simultaneous measurement of dynamic acceleration and strain: (a) Z-axis acceleration and (d) strain.

According to Fig. 6, the prominent peaks have occurred at $7.17 \mathrm{~Hz}$ and $7.14 \mathrm{~Hz}$ in the amplitude spectra of dynamic acceleration and strain measurements, respectively. This value is close to the calculated $1^{\text {st }}$ natural frequency of $8.35 \mathrm{~Hz}$ for the cantilever beam by [43]: 


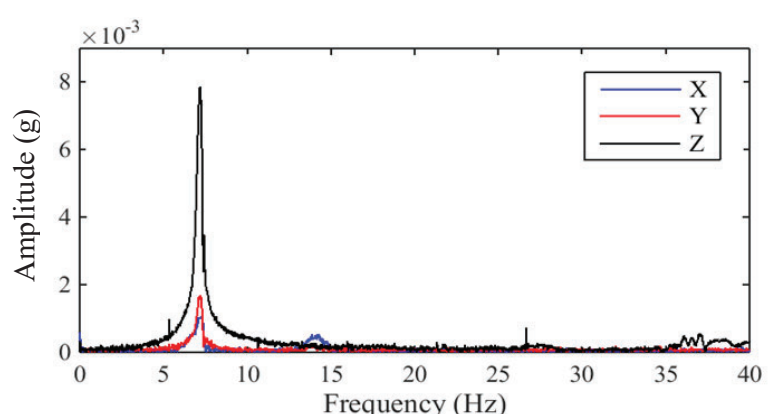

(a)

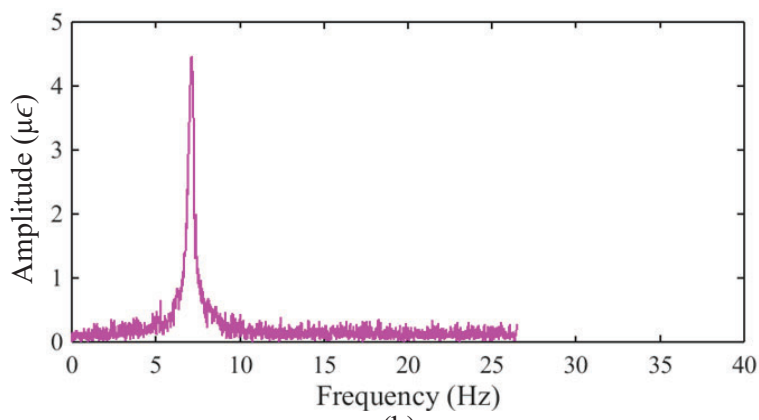

(b)

Fig. 6. Single-sided amplitude spectra of simultaneous: (a) 3-axis dynamic acceleration measurement and (b) dynamic strain measurement.

$$
f_{n}=\frac{\kappa^{2}}{2 \pi} \sqrt{\frac{E I}{\rho A}}
$$

where, $f_{\mathrm{n}}$ is the frequency of $\mathrm{n}^{\text {th }}$ mode $(\mathrm{Hz}), \kappa \approx(2 \mathrm{n}-1) \pi / 2 l$ $\left(\mathrm{m}^{-1}\right), I$ is the moment of inertia $\left(\mathrm{m}^{4}\right), \rho$ is the density $\left(\mathrm{kgm}^{-3}\right)$, and $A$ is the cross section area $\left(\mathrm{m}^{2}\right)$ of the beam. The parameters of structural steel A36 were used for the natural frequency calculation. The existing discrepancy between the theoretical and measured values might be due to an error in the assumption of the cantilever beam and the fix end is not rigid. In the experiment, all tri-axis accelerations are carrying the frequency information as shown in Fig. 6(a). There is measurement noise in the amplitude spectrum of the strain measurement as shown in Fig. 6(b).

\section{COMPARISON WITH COMMERCIAL WIRELESS STRAIN MEASUREMENT SYSTEM}

Simultaneous measurements were acquired with both the MSITA and the CWLSS systems upon cantilever beam mainly to determine the frequency detection accuracy of the MSITA strain measurement results (the acceleration measurements were previously benchmarked with a commercial system in [44]). The strain gauge for CWLSS was attached to the bottom surface at a distance of $0.025 \mathrm{~m}$ from $\mathrm{O}$ (according to Eq. (1) this should provide the same strain value similar to the gauge attached to the top surface). The sampling rate of CWLSS was set to $64 \mathrm{~Hz}$, which was the nearest setting for the sampling rate of $\sim 53 \mathrm{~Hz}$ achieved in the MSITA strain interface. Fig. 7 depicts the time history and the amplitude spectra of an example simultaneous strain measurement result acquired. The dynamic strain from MSITA is almost equal to the signal acquired from the commercial system. The noise level in MSITA is much higher than that of CWLSS. The ADC resolution of the strain interface of MSITA (16 bits) is higher than that of CWLSS (12 bits). Therefore, MSITA tends to pick-up noise than the CWLSS system.

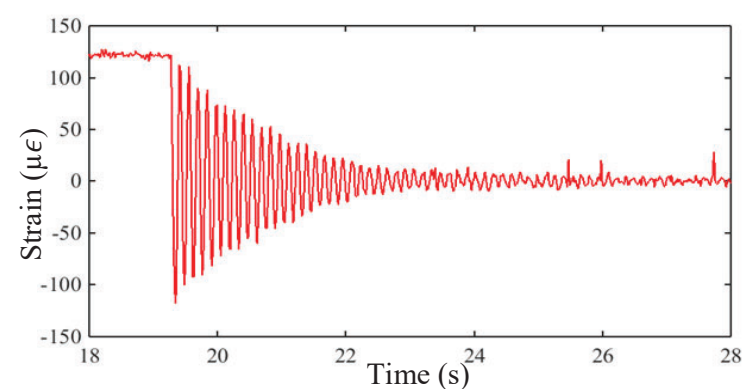

(a)

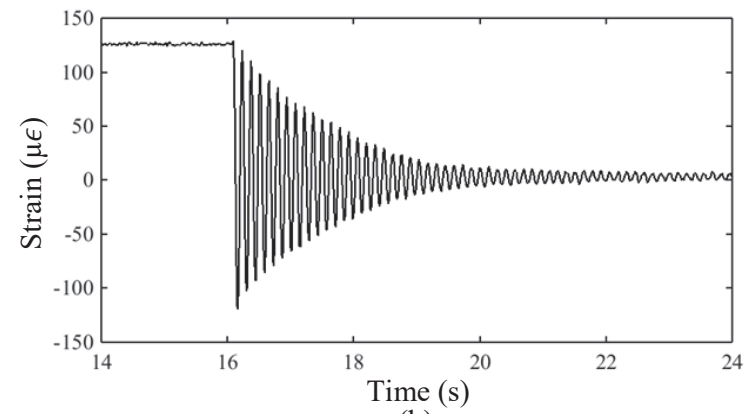

(b)

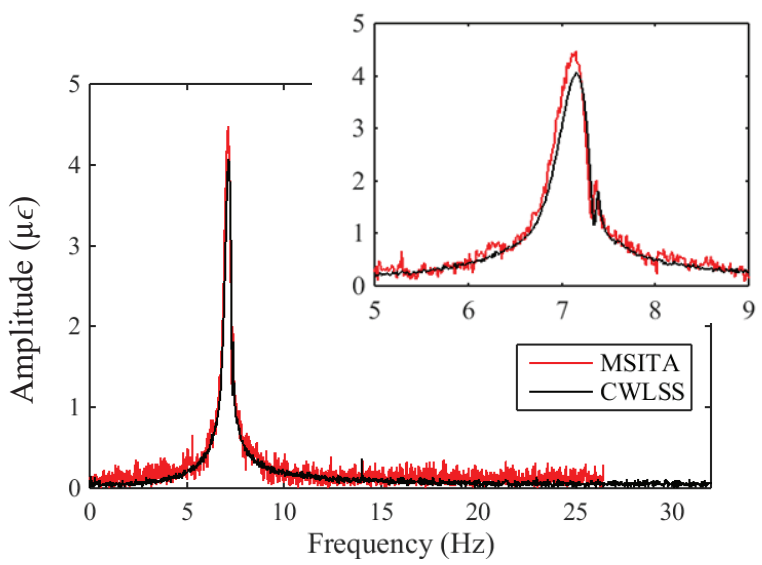

(c)

Fig. 7. Time history of simultaneous strain measurement from: (a) MSITA, (b) CWLSS and (c) single-side amplitude spectra

TABLE III

MAXIMUM VARIATION RANGE OF THE DETERMINED NATURAL FREQUENCY

\begin{tabular}{lccc}
\hline \hline Frequency & \multicolumn{2}{c}{ MSITA } & \multirow{2}{*}{ CWLSS } \\
\cline { 2 - 3 } Determination & Acceleration & Strain & \\
\hline Mode $(\mathrm{Hz})$ & 7.17 & 7.11 & 7.14 \\
\hline Difference $(\mathrm{mHz})$ & & $-60 /-30$ & \\
\hline \hline
\end{tabular}




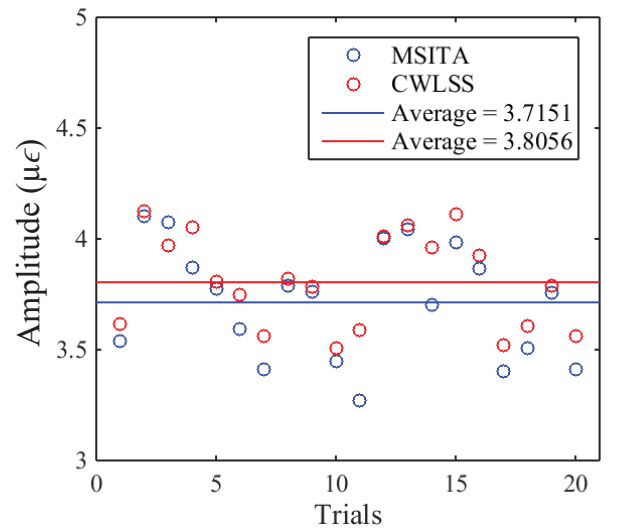

Fig. 8. Amplitude comparison of determined natural frequency between MSITA and CWLSS

The identified natural frequencies from 20 strain measurements from those two systems were compared. In the case of MSITA, the Z-axis acceleration results were considered as it provided the opportunity to compare the natural frequency determination between simultaneously measured acceleration and strain. The measurement results of both systems were denoised using wavelet denoising primarily to achieve prominent peak smoothing of the amplitude spectra of the MSITA in order to overcome peak ambiguity generated by higher noise level. The denoising of the measurement results from CWLSS was done only to achieve a fair comparison between the two systems.

The mode of the determined natural frequency values of the denoised measurement was considered as it provides the ability of the systems to repeat the information, and those modes and difference values are given in Table III. The natural frequency determined by strain measurement of MSITA is $60 \mathrm{mHz}$ less than that by the acceleration measurement. However, when considering the repeatability of natural frequency determination, this value can be stated as MSITA system quality for the current configuration given in Table I. The natural frequency determination from the strain measurement of MSITA has a $-30 \mathrm{mHz}$ error when benchmarked with the CWLSS system. These measurements had a frequency resolution $16 \mathrm{mHz}$.

Fig. 8 provides the comparison of amplitude of the determined natural frequency in the spectra from simultaneous strain measurement results of the cantilever beam by MSITA and CWLSS. The amplitudes of single-sided amplitude spectra of strain measurement results by MSITA for each trial have good agreement with those from CWLSS. According to Fig. 8, the strain sensitivity of the RFID-based wireless system is $97.6 \%$ of that of CWLSS system considering average amplitude values of all trials. This can also be visualized in Fig. 7 in which both systems show almost the same dynamic strain signal amplitude. Less sensitivity of MSITA shown in the amplitude comparison is predominantly due to the ambient noise present which tends to suppress the peak amplitude.

\section{CONCLUSION}

In this paper, a wireless multi-sensor system to measure dynamic acceleration and strain of infrastructure was developed with a custom designed multi-sensor integrated semi-passive tag antenna which could even be mounted on civil structures made out of metal. Design considerations for dynamic measurements under constraints imposed by the operational states of the RFID tag chip and the data acquisition capabilities of the RFID reader were discussed. The sensor system could measure simultaneous dynamic 3axis acceleration and strain of infrastructure to achieve $40-\mathrm{Hz}$ and $26.5-\mathrm{Hz}$ spectral bandwidth, respectively. The measurements were conducted using a cantilever steel beam, and the results carry the natural frequency information of the beam. The measurement results were further supported by comparing the developed system's ability to determine the natural frequency of the cantilever beam and that of a commercial wireless strain measurement system. The difference between the natural frequency determination from dynamic acceleration and strain was $60 \mathrm{mHz}$ for the developed system. There was a minimal error of $30 \mathrm{mHz}$ in natural frequency representation by the developed system when benchmarked against a commercial wireless strain measurement system. Therefore, the proposed RFID-based wireless multi-sensory system has the potential to be used for multiple measurand-based infrastructure health monitoring.

\section{ACKNOWLEDGEMENT}

The authors would like to acknowledge the indispensable intellectual contribution of late Professor Sergey Kharkovsky and the valued technical services of Mr. Ranjith Ratnayake of the Infrastructure Health Monitoring Lab, Western Sydney University, Australia.

\section{REFERENCES}

[1] T. Chan and D. P. Thambiratnam, Engineering Tools, Techniques and Tables: Structural Health Monitoring in Australia. Hauppauge, US: Nova, 2011.

[2] J. A. Rice and B. F. Spencer Jr, "Flexible smart sensor framework for autonomous full-scale structural health monitoring," Newmark Structural Engineering Laboratory. University of Illinois at UrbanaChampaign. 1940-9826, 2009.

[3] S. Kim, S. Pakzad, D. Culler, J. Demmel, G. Fenves, S. Glaser, et al., "Health monitoring of civil infrastructures using wireless sensor networks," in Proc. 6th int. conf. Inform. Process. sensor networks, 2007, pp. 254-263.

[4] P. L. Jerome, W. Yang, J. L. Kenneth, Y. Jin-Hak, and Y. ChungBang, "Performance monitoring of the Geumdang Bridge using a dense network of high-resolution wireless sensors," Smart Mater. Struct., vol. 15, no. 6, pp. 1561-1575, 2006.

[5] M. J. Whelan, M. V. Gangone, and K. D. Janoyan, "Highway bridge assessment using an adaptive real-time wireless sensor network," IEEE Sens. J., vol. 9, no. 11, pp. 1405-1413, 2009.

[6] S. Jang, H. Jo, S. Cho, K. Mechitov, J. A. Rice, S. H. Sim, et al., "Structural health monitoring of a cable-stayed bridge using smart sensor technology: deployment and evaluation," Smart Struct. Syst., vol. 6, no. 5-6, pp. 439-459, 2010.

[7] S. Zerbst, G. Haake, J. Reetz, J. Lynch, and R. Rolfes, "Integral SHM-system for offshore wind turbines using smart wireless sensors," in Proc. 6th Int. Workshop Struct. Health Monit., 2007, pp. 1889-1896.

[8] A. T. Zimmerman, M. Shiraishi, R. A. Swartz, and J. P. Lynch, "Automated Modal Parameter Estimation by Parallel Processing 
within Wireless Monitoring Systems," J. Infrastruct. Syst., vol. 14, no. 1, pp. 102-113, 2008.

[9] H. Murayama, D. Wada, and H. Igawa, "Structural health monitoring by using fiber-optic distributed strain sensors with high spatial resolution," Photonic Sens., vol. 3, no. 4, pp. 355-376, 2013.

[10] S. Fabio Luis Marques dos, P. Bart, L. Jenny, D. Wim, and G. Luiz Carlos Sandoval, "The use of strain gauges in vibration-based damage detection," J. Physics: Conf. Series, vol. 628, no. 1, p. 012119, 2015.

[11] A. Philips Adewuyi, Z. Wu, and N. H. M. Kammrujaman Serker, "Assessment of vibration-based damage identification methods using displacement and distributed strain measurements," Struct. Health Monit., vol. 8, no. 6, pp. 443-461, 2009.

[12] M. Aldo, C. Agnese, P. Salvatore, B. Romeo, and Z. Luigi, "Moda analysis of a cantilever beam by use of Brillouin based distributed dynamic strain measurements," Smart Mater. Struct., vol. 21, no. 12, p. $125022,2012$.

[13] A. Panopoulou, D. Roulias, T. H. Loutas, and V. Kostopoulos, "Health monitoring of aerospace structures using fibre Bragg gratings combined with advanced signal processing and pattern recognition techniques," Strain, vol. 48, no. 3, pp. 267-277, 2012.

[14] S. H. Sim, B. F. S. Jr., and T. Nagayama, "Multimetric sensing for structural damage detection," J. Eng. Mech., vol. 137, no. 1, pp. 22 $30,2011$.

[15] E. M. Mugambi, K. Kwan, B. C. Laskowski, T. K. Ooi, and A. Corder, "MEMS-based strain and corrosion sensors for structural health monitoring," in Proc. 6th Int. Workshop Struct. Health Monit, Stanford University, Stanford, Calif., 2007.

[16] S. H. Sim and J. B. F. Spencer. (2015). Combined use of acceleration and strain in damage detection. Available: https://www.researchgate.net/publication/266587628.

[17] C. Haksoo, C. Sukwon, and C. Hojung, "Structural health monitoring system based on strain gauge enabled wireless sensor nodes," in 5 th Int. Conf. Networked Sensing Syst., 2008, pp. 211-214.

[18] P. L. Jerome, W. Yang, J. L. Kenneth, Y. Jin-Hak, and Y. ChungBang, "Performance monitoring of the Geumdang Bridge using a dense network of high-resolution wireless sensors," Smart Mater Struct., vol. 15, no. 6, pp. 1561-1575, 2006.

[19] A. C. L. Wong, P. A. Childs, and G.-D. Peng, "Multiplexed fibre Fizeau interferometer and fire Bragg grating sensor system for simultaneous measurement of quasi-static strain and temperature using discrete wavelet transformation," Meas. Sci. Technol., vol. 17, no. 2, pp. 384-392, 2006.

[20] H.-K. Kang, H.-J. Bang, C.-S. Hong, and C.-G. Kim, "Simultaneous measurement of strain, temperature and vibration frequency using fibre optic sensor," Meas. Sci. Technol., vol. 13, pp. 1191-1196, 2002.

[21] D.-D. Ho, K.-D. Nguyen, H.-S. Yoon, and J.-T. Kim, "Multiscale acceleration-dynamic strain-impedance sensor system for structural health monitoring," Int. J. Distrib. Sens. Netw., vol. 8, no. 10, pp. 1$17,2012$.

[22] D.-D. Ho, K.-D. Nguyen, H.-S. Yoon, and J.-T. Kim, "Multiscale acceleration-dynamic strain-impedance sensor system for structural health monitoring," Int. J. Distrib. Sens. Netw., vol. 8, no. 10, 2012.

[23] F. Federici, R. Alesii, A. Colarieti, F. Graziosi, and M. Faccio, "Design and validation of a wireless sensor node for long term structural health monitoring," in IEEE Sens., 2013, pp. 1-4.

[24] A. Ruhanen, M. Hanhikorpi, F. Bertuccelli, A. Colonna, W. Malik, D. Ranasinghe, et al., "Sensor-enabled RFID tag handbook," BRIDGE, IST-2005-033546, January, 2008

[25] N. C. Karmakar, S. M. Roy, S. Jenvey, S. Preradovic, T. D. Vo, and G. F. Swiegers, "Development of a low-cost backscattered semiactive RFID tag at $2.4 \mathrm{GHz}$," Int. J. RF Microwave Comput. Aided Eng., vol. 17, no. 6, pp. 574-582, 2007.

[26] C. Occhiuzzi, C. Paggi, and G. Marrocco, "Passive RFID strainsensor based on meander-line antennas," IEEE Trans. Antenna Propag, vol. 59, no. 12, pp. 4836-4840, 2011.

[27] X. Yi, T. Wu, Y. Wang, R. T. Leon, M. M. Tentzeris, and G. Lantz, "Passive wireless smart-skin sensor using RFID-based folded patch antennas," Int. J. Smart Nano Mater., vol. 2, no. 1, pp. 22-38, 2011.

[28] R. Bhattacharyya, C. Floerkemeier, and S. Sarma, "Towards tag antenna based sensing - An RFID displacement sensor," in IEEE Int. Conf. RFID, 2009, pp. 95-102.

[29] S. Caizzone, E. DiGiampaolo, and G. Marrocco, "Wireless crack monitoring by stationary phase measurements from coupled RFID tags," IEEE Trans. Antennas Propag., vol. 62, no. 12, pp. 6412-6419, 2014.
[30] S. Ogawa and T. Sato, "Monitoring of concrete structures using passive type RFID tags with sensory functions," in Int. Conf. Elect. Eng., Okinawa, Japan, 2008.

[31] Y. Ikemoto, S. Suzuki, H. Okamoto, H. Murakami, X. Lin, H. Itoh, et al., "Force sensor system for structural health monitoring using passive RFID tags for structural health monitoring," in PORTABLEPOLYTRONIC 2008 - 2nd IEEE Int. Interdisciplinary Conf. Portable Inform. Devices and 2008 7th IEEE Conf. Polymers and Adhesives Microelectronics Photonics, 2008, pp. 1-6.

[32] W. Leon-Salas, S. Kanneganti, and C. Halmen, "Development of a smart RFIDbased corrosion sensor," in IEEE Sens., 2011, pp. 534537.

[33] S. Manzari, T. Musa, M. Randazzo, Z. Rinaldi, A. Meda, and G. Marrocco, "A passive temperature radio-sensor for concrete maturation monitoring," in IEEE RFID Technol. and Appl. Conf. (RFID-TA), 2014, pp. 121-126.

[34] P. Pursula, I. Marttila, K. Nummila, and H. Seppä, "High frequency and ultrahigh frequency radio frequency identification passive sensor transponders for humidity and temperature measurement within building structures," IEEE Trans. Instrum. Meas., vol. 62, no. 9, pp. 2559-2566, 2013.

[35] E. D. Giampaolo, A. DiCarlofelice, and A. Gregori, "An RFIDenabled wireless strain gauge sensor for static and dynamic structural monitoring," IEEE Sens. J., vol. 17, no. 2, pp. 286-294, 2016.

[36] RFID Journal, (2015). RFID helps contractors to measure strain during construction of Seattle rail tunnels. Available: http://www.rfidjournal.com/articles/view?12913, accessed: 10 September 2015

[37] D. D. Donno, L. Catarinucci, and L. Tarricone, "RAMSES: RFID augmented module for smart environmental sensing," IEEE Trans. Instrum. Meas., vol. 63, no. 7, pp. 1701-1708, 2014.

[38] A. P. Sample and J. R. Smith, "The wireless identification and sensing platform," in Wirelessly Powered Sensor Networks and Computational RFID, J. R. Smith, Ed., ed New York, NY, USA: Springer Science + Business Media, 2013, pp. 33-56.

[39] D. Jayawardana, S. Kharkovsky, and R. Liyanapathirana, "Construction material mountable UHF RFID tag antenna," Microwave Opt. Technol. Lett., vol. 58, no. 9, pp. 2231-2237, September 2016

[40] J. Pople, "Errors and uncertainties of strain measurement," in Strain gauge technology, A. L. Window, Ed., 2nd ed Crown House, Linton Road, Barking, Essex IG118JU, England: Elsevier Science Publishers LTD, pp. 217-272.

[41] Texas Instruments, (2015). OPAx333 1.8-V, micropower, CMOS operational amplifiers, zero-drift series (Rev. E) Available: http://www.ti.com/product/opa333?keyMatch=opa333\&tisearch=Sear ch-ENEverything, accessed: 20 September 2015.

[42] Impinj, (2013). Monza X-8K Dura Datasheet, [Revision 1.1]. Available: https://support.impinj.com/hc/en-us/articles/202756868Monza-X-8K-DuraDatasheet, accessed: 1 May 2014.

[43] R. G. Merritt, "Vibration of systems having distributed mass and elasticity," in Harris' Shock and Vibration Handbook, A. G. Piersol and T. L. Paez, Eds., 6th ed New York, NY, USA: McGraw-Hill, 2010, pp. $19-25$

[44] D. Jayawardana, S. Kharkovsky, R. Liyanapathirana, and X. Zhu, "Measurement system with accelerometer integrated RFID tag for infrastructure health monitoring," IEEE Trans. Instrum. Meas., vol. 65, no. 5, pp. 1163-1171, May 2016.

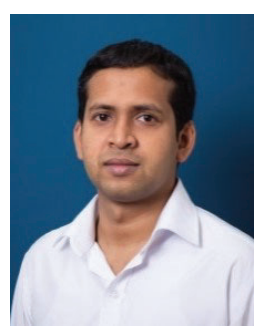

Devaka Jayawardana (S'15-M'17) was born in Matara, Sri Lanka, in 1984. He received B.Sc. degree in electronics and telecommunication engineering in 2009 and P.G.Dip. in electronics and automation in 2013 from University of Moratuwa, Moratuwa, Sri Lanka. He received Ph.D. degree in infrastructure engineering in 2017 from Western Sydney University, Penrith, Australia. From 2009 to 2010, he worked as a Research Engineer in Arthur C. Clarke Institute for Modern Technologies, Sri Lanka. He was with the Ceylon 
Electricity Board, Sri Lanka, both as a Transmission Planning Engineer and a Communication Systems Engineer in the periods of 2010 to 2011 and 2011 to 2014, respectively. Dr. Jayawardana currently works as a Research and Development Engineer for Ceylon Electricity Board, Sri Lanka. His current research interests include radio frequency identification (RFID), low power wireless measurement system design, structural health monitoring, e-mobility and dynamic wireless power transfer.

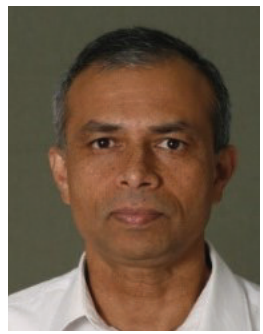

Ranjith Liyanapathirana (M'89, SM'15) received Ph.D. in electrical engineering from Memorial University of Newfoundland, Canada in 1995. He was an Associate Lecturer and Research Fellow at the University of Western Australia, Perth (1994-1998). In 1998 he joined Western Sydney University as Lecturer in Telecommunications. He is currently a Senior Lecturer in the School of Computing, Engineering and Mathematics there. His current research interests are wireless communications, infrastructure health monitoring, and sensor applications in biomedical engineering. Dr. Liyanapathirana has published over 150 articles in peer-reviewed international conferences, IEEE/IET and other Journals. He is a senior member of the Australian Computer Society (ACS).

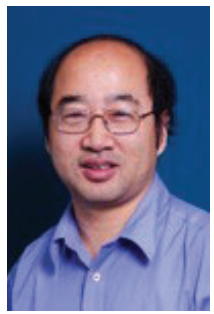

Xinqun Zhu received the B.Eng., M.Sc. and Ph.D. degrees from Zhejiang Sci-Tech University in 1987, Southwest Jiaotong University in 1990 and the Hong Kong Polytechnic University in 2001. $\mathrm{He}$ is currently an Associate Professor in Structural Engineering at University of Technology, Sydney, Australia.

Dr. Zhu is an Associate Editor of the International Journal of Advances in Structural Engineering. He has published two books and one book chapter as well as 85 refereed archival journal articles. His research interests are structural health monitoring and condition assessment, advanced signal processing and smart sensor technology. 